\title{
Inference for the Geometric Extreme Exponential Distribution under Progressive Type II Censoring
}

\author{
Reza Pakyari \\ Department of Mathematics, Faculty of Science, Arak University, Arak 38156-8-8349, Iran \\ Correspondence should be addressed to Reza Pakyari, r-pakyari@araku.ac.ir
}

Received 22 June 2012; Accepted 11 July 2012

Academic Editors: M. Galea and M. Montero

Copyright (C) 2012 Reza Pakyari. This is an open access article distributed under the Creative Commons Attribution License, which permits unrestricted use, distribution, and reproduction in any medium, provided the original work is properly cited.

Geometric extreme exponential (GE-exponential) is one of the nonnegative right-skewed distribution that is suitable for analyzing lifetime data. It is well known that the maximum likelihood estimators (MLEs) of the parameters lead to likelihood equations that have to be solved numerically. In this paper, we provide explicit estimators through an approximation of the likelihood equations based on progressively Type-II-censored samples. The approximate estimators are then used as starting values to find the MLEs numerically. The bias and variances of the MLEs are calculated for a wide range of sample sizes and different progressive censoring schemes through a Monte Carlo simulation study. Moreover, formulas for the observed Fisher information are given which could be used to construct asymptotic confidence intervals. The coverage probabilities of the confidence intervals and the percentage points of pivotal quantities associated with the MLEs are also calculated. A real dataset has been studied for illustrative purposes.

\section{Introduction}

Progressive censoring is one of the important sampling techniques that was first introduced by Herd [1] and its importance in life testing reliability experiments was discussed by Cohen [2].

The progressive Type-II censoring is as follows. Suppose $n$ units are placed on test. At the time of the first failure, $R_{1}$ units are randomly removed from the $n-1$ surviving units. Next, at the time of the second failure, $R_{2}$ units are randomly removed from the $n-R_{1}-2$ surviving units, and so on. Finally, after the $m$ th failure, all remaining $R_{m}$ units are removed. Thus, we observe $m$ complete failures and $R_{1}+R_{2}+\cdots+R_{m}$ items are progressively censored from the $n$ units under test, and so $n=m+\left(R_{1}+R_{2}+\cdots+R_{m}\right)$. The vector $R=\left(R_{1}, \ldots, R_{m}\right)$ is called the censoring scheme and is fixed prior to the study. If $R=(0, \ldots, 0)$, no withdrawals are made which correspond to the complete sample and the ordinary order statistics will 
be obtained. If $R=(0, \ldots, 0, n-m)$, we obtain the conventional Type-II right censoring. We will denote the progressive Type-II censored data by $X_{1: m: n}<X_{2: m: n}<\cdots<X_{m: m: n}$. For an excellent discussion on progressive Type-II censoring technique see the monograph of Balakrishnan and Aggarwala [3] and the recent discussion paper by Balakrishnan [4].

There are numerous articles in the literature dealing with inferential procedures based on the progressively Type-II censoring data for a wide variety of lifetime distributions. See for example Mann [5, 6], Balakrishnan et al. [7], Balakrishnan and Kannan [8], Balakrishnan et al. [9], Kim and Han [10], Lin et al. [11], and Asgharzadeh [12] among others who study the inferential procedures for the Weibull, extreme value, logistic, normal, half-logistic, loggamma, and generalized exponential models, respectively.

GE-exponential distribution was introduced by Marshal and Olkin [13] and further studied extensively by Adamidis and Loukas [14] and Adamidis et al. [15]. Recently, this model was of interest of authors as a natural competitor to the other well-established lifetime models such as Weibull, lognormal, and gamma distributions (see, e.g. [16, 17]).

In this paper, we study the inference for the parameters of the GE-exponential distribution based on progressively Type-II censored data.

The rest of the paper is organized as follows. The MLEs of the parameters are studied in Section 2. In Section 3, we provide an approximation to the likelihood function which leads to explicit approximate estimators of the parameters. The observed Fisher information is studied in Section 4. The performance of the maximum likelihood estimators is evaluated in terms of bias and variance through a large number of Monte Carlo simulations and the results are given in Section 5. Using the asymptotic normality of the proposed estimators, the coverage probabilities of the asymptotic confidence intervals and the percentage points for the pivotal quantities are calculated by simulations and the results are provided in Section 6. Finally, Section 7 is devoted to study a real dataset for illustration of the proposed methodology.

\section{Maximum Likelihood Estimation}

Let the failure times follow a geometric extreme exponential distribution with shape parameter $\gamma$ and scale parameter $\lambda$ and with probability density function:

$$
f(x ; \lambda, \gamma)=\frac{\gamma \lambda e^{-\lambda x}}{\left(1-\bar{\gamma} e^{-\lambda x}\right)^{2}}, \quad(x>0),
$$

where $\bar{\gamma}=1-\gamma$. The cumulative distribution function is give by

$$
F(x ; \lambda, \gamma)=\frac{1-e^{-\lambda x}}{1-\bar{\gamma} e^{-\lambda x}}
$$

The GE-exponential reduces to the exponential distribution if we take $\gamma=1$. When $0<\gamma<1$, the distribution reduces to exponential geometric distribution studied by Adamidis and Loukas [14]. The GE-exponential distribution has decreasing failure rate when $0<\gamma<1$ 
and increasing failure rate when $\gamma \geq 1$. The density function is unimodal with modal value equal to $-\lambda \gamma\{4 \bar{\gamma}\}^{-1}$ when $\gamma>2$. Hereafter, we assume $\gamma \geq 1$.

Let $X_{1: m: n}, \ldots, X_{m: m: n}$ be a progressively Type-II censored sample from density function (2.1). The likelihood function based on the censored sample is given by (see [3])

$$
\begin{aligned}
L(\lambda, \gamma) & =C \prod_{i=1}^{m} \frac{\gamma \lambda e^{-\lambda x_{i}}}{\left(1-\bar{\gamma} e^{-\lambda x_{i}}\right)^{2}}\left\{1-\frac{1-e^{-\lambda x_{i}}}{1-\bar{\gamma} e^{-\lambda x_{i}}}\right\}^{R_{i}} \\
& =C(\gamma \lambda)^{m} \prod_{i=1}^{m} \frac{\gamma^{R_{i}} e^{-\lambda\left(R_{i}+1\right) x_{i}}}{\left(1-\bar{\gamma} e^{-\lambda x_{i}}\right)^{R_{i}+2}},
\end{aligned}
$$

where

$$
C=n\left(n-1-R_{1}\right)\left(n-2-R_{1}-R_{2}\right) \cdots\left(n-m+1-R_{1}-\cdots-R_{m-1}\right) .
$$

The log-likelihood function may be written as

$$
\begin{aligned}
L^{*}(\lambda, \gamma)= & K+m \log \gamma+m \log \lambda+\log \gamma \sum_{i=1}^{m} R_{i}-\lambda \sum_{i=1}^{m}\left(R_{i}+1\right) x_{i} \\
& -\sum_{i=1}^{m}\left(R_{i}+2\right) \log \left(1-\bar{\gamma} e^{-\lambda x_{i}}\right)
\end{aligned}
$$

where $K$ is a constant. Let $z_{i}=\lambda x_{i}$, now the log-likelihood function may be rewritten as

$$
\begin{aligned}
L^{*}(\lambda, \gamma)= & K+m \log \gamma+m \log \lambda+\log \gamma \sum_{i=1}^{m} R_{i}-\sum_{i=1}^{m}\left(R_{i}+1\right) z_{i} \\
& -\sum_{i=1}^{m}\left(R_{i}+2\right) \log \left(1-\bar{\gamma} e^{-z_{i}}\right)
\end{aligned}
$$

The likelihood equations are then given by

$$
\begin{gathered}
\frac{\partial}{\partial \lambda} L^{*}(\lambda, \gamma)=\frac{m}{\lambda}-\frac{1}{\lambda} \sum_{i=1}^{m}\left(R_{i}+1\right) z_{i}-\frac{1}{\lambda} \sum_{i=1}^{m}\left(R_{i}+2\right)\left(\frac{\bar{\gamma} z_{i} e^{-z_{i}}}{1-\bar{\gamma} e^{-z_{i}}}\right)=0, \\
\frac{\partial}{\partial \gamma} L^{*}(\lambda, \gamma)=\frac{m}{\gamma}+\frac{\sum_{i=1}^{m} R_{i}}{\gamma}-\sum_{i=1}^{m}\left(R_{i}+2\right)\left(\frac{e^{-z_{i}}}{1-\bar{\gamma} e^{-z_{i}}}\right)=0,
\end{gathered}
$$

which may be simplified as

$$
\begin{gathered}
m-\sum_{i=1}^{m}\left(R_{i}+1\right) z_{i}-\sum_{i=1}^{m}\left(R_{i}+2\right)\left(\frac{\bar{\gamma} z_{i} e^{-z_{i}}}{1-\bar{\gamma} e^{-z_{i}}}\right)=0, \\
m+\sum_{i=1}^{m} R_{i}-\gamma \sum_{i=1}^{m}\left(R_{i}+2\right)\left(\frac{e^{-z_{i}}}{1-\bar{\gamma} e^{-z_{i}}}\right)=0 .
\end{gathered}
$$


The MLEs of the parameters $\lambda$ and $\gamma$ are the solutions of the system of nonlinear (2.9) and (2.10) which cannot be expressed explicitly. Standard numerical procedures such as Newton's method needed to solve the above system of nonlinear equations. However, as is necessary for every numerical method, are starting values are needed to implement the numerical procedure. In the following section, we present an approximate MLEs for the parameters $\lambda$ and $\gamma$ to be used as starting values for implementation of the Newton's method to solve the system of nonlinear (2.9) and (2.10). The approximation method is based on first finding a moment estimator for the shape parameter $\gamma$ and based on this estimator finding an approximate MLE of the scale parameter $\lambda$ and using that to find an approximate MLE of $\gamma$ by either (2.9) or (2.10).

\section{Approximate Maximum Likelihood Estimators}

As was studied in the previous section, the maximum likelihood estimators of the parameters are given only implicitly by solving the system of nonlinear equations (2.9) and (2.10) by an appropriate numerical method. In this section, we provide approximate maximum likelihood estimators to be used as starting values to solve the system of nonlinear equations numerically. We propose a two-step approximation method, in which the moment estimator of the shape parameter $\gamma$ is used to find the approximate maximum likelihood estimator of the scale parameter $\lambda$. The approximate MLE of $\gamma$ is then can be found by either the likelihood equations (2.9) or (2.10) and the value of the approximate MLE of $\lambda$.

To find a moment estimator of $\gamma$, we consider the coefficient of variation (C.V.) which is given by

$$
\text { C.V. }=-\frac{\bar{\gamma}\left\{2 \Phi(\bar{\gamma}, 2,1)-\left(\gamma \ln ^{2} \gamma\right) / \bar{\gamma}^{2}\right\}^{1 / 2}}{\sqrt{\gamma} \ln \gamma},
$$

where $\Phi(a, b, c)$ is the Lerch's transcendent (see, for example, [18]).

Note that C.V. is independent from the scale parameter $\lambda$ and depends only on the shape parameter $\gamma$. Table 1 provides the value of the C.V. for different values of the shape parameter $\gamma$. This table may be used to find the moment estimator of the shape parameter $\gamma$ for the given value of the sample C.V. when the shape parameter is unknown.

Let $X_{1: m: n}, \ldots, X_{m: m: n}$ be a progressively Type-II censored sample from $f(x ; \lambda, \tilde{\gamma})$, where $\tilde{\gamma}$ is the moment estimator of $\gamma$. The likelihood equation (2.9) may now be rewritten in terms of $\tilde{\gamma}$ as

$$
m-\sum_{i=1}^{m}\left(R_{i}+1\right) z_{i}-\sum_{i=1}^{m}\left(R_{i}+2\right)\left(\frac{\overline{\tilde{\gamma}} z_{i} e^{-z_{i}}}{1-\overline{\tilde{\gamma}} e^{-z_{i}}}\right)=0,
$$

where $\overline{\tilde{\gamma}}=1-\tilde{\gamma}$. The MLE of the scale parameter $\lambda$ is the solution of the nonlinear equation (3.2) which can not be expressed explicitly. 
Let us approximate the likelihood equation (3.2) by approximating the function $h(z)=$ $e^{-z} /\left(1-\overline{\tilde{\gamma}} e^{-z}\right)$ in a Taylor series expansion around $E\left(Z_{i: m: n}\right)=v_{i: m: n}$. From Balakrishnan and Sandhu [19] we have

$$
F\left(Z_{i: m: n}\right)=U_{i: m: n}
$$

where $U_{i: m: n}$ is the $i$ th order statistic of a progressively Type-II censored sample from the $U$ $(0,1)$ distribution.

We then have

$$
Z_{i: m: n}=F^{-1}\left(U_{i: m: n}\right)=\log \left(\frac{\overline{\tilde{r}} U_{i: m: n}-1}{U_{i: m: n}-1}\right),
$$

and hence

$$
v_{i: m: n}=E\left(Z_{i: m: n}\right)=\log \left(\frac{\overline{\tilde{\gamma}} \alpha_{i: m: n}-1}{\alpha_{i: m: n}-1}\right),
$$

where $\alpha_{i: m: n}=E\left(U_{i: m: n}\right)$ and

$$
\alpha_{i: m: n}=1-\prod_{j=m-i+1}^{m} \frac{j+R_{m-j+1}+\cdots+R_{m}}{j+1+R_{m-j+1}+\cdots+R_{m}}, \quad i=1, \ldots, m .
$$

Expanding $h\left(z_{i}\right)$ around $v_{i: m: n}$ by Taylor series expansion and keeping the first two terms, we deduce

$$
\begin{aligned}
h\left(z_{i}\right) & \approx h\left(v_{i: m: n}\right)+\left.h^{\prime}\left(z_{i}\right)\right|_{z=v_{i: m: n}}\left(z_{i}-v_{i: m: n}\right) \\
& =\alpha_{i}+\beta_{i} z_{i}
\end{aligned}
$$

where

$$
\begin{gathered}
\alpha_{i}=\frac{e^{-v_{i: m: n}}\left(1-\overline{\tilde{\gamma}} e^{-v_{i: m: n}}+v_{i: m: n}\right)}{\left(1-\overline{\tilde{\gamma}} e^{-v_{i: m: n}}\right)^{2}}, \\
\beta_{i}=\frac{-e^{-v_{i: m: n}}}{\left(1-\overline{\tilde{\gamma}} e^{-v_{i: m: n}}\right)^{2}} .
\end{gathered}
$$

Therefore, the likelihood equation (3.2) may be approximated by

$$
m-\sum_{i=1}^{m}\left(R_{i}+1\right) z_{i}-\overline{\tilde{\gamma}} \sum_{i=1}^{m}\left(R_{i}+2\right) z_{i}\left(\alpha_{i}+\beta_{i} z_{i}\right)=0 .
$$


Equation (3.9) may be rewritten as

$$
\left\{\overline{\widetilde{r}} \sum_{i=1}^{m}\left(R_{i}+2\right) \beta_{i} x_{i}^{2}\right\} \lambda^{2}+\left\{\overline{\widetilde{\gamma}} \sum_{i=1}^{m}\left(R_{i}+2\right) \alpha_{i} x_{i}+\sum_{i=1}^{m}\left(R_{i}+1\right) x_{i}\right\} \lambda-m=0
$$

or

$$
A \lambda^{2}+B \lambda-m=0
$$

where

$$
\begin{gathered}
A=\overline{\widetilde{\gamma}} \sum_{i=1}^{m}\left(R_{i}+2\right) \beta_{i} x_{i}^{2}, \\
B=\overline{\tilde{\gamma}} \sum_{i=1}^{m}\left(R_{i}+2\right) \alpha_{i} x_{i}+\sum_{i=1}^{m}\left(R_{i}+1\right) x_{i} .
\end{gathered}
$$

Equation (3.11) is a quadratic equation in terms of $\lambda$ with the following two solutions:

$$
\frac{-B \pm \sqrt{B^{2}+4 A m}}{2 A} .
$$

However, one of the solutions is inadmissible since $A>0$. Hence, the approximate MLE of the scale parameter $\lambda$ is given explicitly by

$$
\breve{\lambda}=\frac{-B+\sqrt{B^{2}+4 A m}}{2 A} .
$$

The approximate MLE of $\gamma$, say $\breve{\gamma}$ is then maybe found by plugging in the value of $\breve{\lambda}$ in either (2.9) or (2.10) and solve the equation by a numerical method. Once the values of $\breve{\lambda}$ and $\breve{\gamma}$ are found, we could use them as good starting values to solve the likelihood (2.9) and (2.10) numerically in order to find the MLEs of $\lambda$ and $\gamma$.

\section{Fisher Information}

In this section, we derive the asymptotic variance-covariance matrix of the maximum likelihood estimators of the parameters $\lambda$ and $\gamma$.

The observed Fisher information may be obtained from the likelihood equations (2.7) and (2.8) as

$$
I=\left[\begin{array}{ll}
I_{11} & I_{12} \\
I_{21} & I_{22}
\end{array}\right]
$$


where

$$
\begin{gathered}
I_{11}=-\frac{\partial^{2}}{\partial \lambda^{2}} L^{*}=\frac{m}{\lambda^{2}}-\frac{1}{\lambda^{2}} \sum_{i=1}^{m}\left(R_{i}+2\right) \frac{\bar{\gamma} z_{i}^{2} e^{-z_{i}}}{\left(1-\bar{\gamma} e^{-z_{i}}\right)^{2}}, \\
I_{12}=I_{21}=-\frac{\partial^{2}}{\partial \lambda \partial \gamma} L^{*}=-\frac{1}{\lambda} \sum_{i=1}^{m}\left(R_{i}+2\right) \frac{z_{i} e^{-z_{i}}}{\left(1-\bar{\gamma} e^{-z_{i}}\right)^{2}}, \\
I_{22}=-\frac{\partial^{2}}{\partial \gamma^{2}} L^{*}=\frac{m}{\gamma^{2}}+\frac{\sum_{i=1}^{m} R_{i}}{\gamma^{2}}-\sum_{i=1}^{m}\left(R_{i}+2\right) \frac{e^{-2 z_{i}}}{\left(1-\bar{\gamma} e^{-z_{i}}\right)^{2}} .
\end{gathered}
$$

The asymptotic variance-covariance matrix of the MLEs is then given by inverting the observed Fisher information matrix:

$$
V(\widehat{\lambda}, \widehat{\gamma})=\left[\begin{array}{ll}
I_{11} & I_{12} \\
I_{12} & I_{22}
\end{array}\right]^{-1}=\left[\begin{array}{ll}
I^{11} & I^{12} \\
I^{12} & I^{22}
\end{array}\right]
$$

where

$$
I^{11}=\frac{I_{22}}{I_{11} I_{22}-I_{12}^{2}}, \quad I^{12}=\frac{-I_{12}}{I_{11} I_{22}-I_{12}^{2}}, \quad I^{22}=\frac{I_{11}}{I_{11} I_{22}-I_{12}^{2}} .
$$

These quantities are estimated by evaluating them at MLEs

$$
\widehat{I}^{11}=\left.\frac{I_{22}}{I_{11} I_{22}-I_{12}^{2}}\right|_{\lambda=\widehat{\lambda}, r=\widehat{\gamma}}, \quad \widehat{I}^{12}=\left.\frac{-I_{12}}{I_{11} I_{22}-I_{12}^{2}}\right|_{\lambda=\widehat{\lambda}, \gamma=\widehat{\gamma}}, \quad \widehat{I}^{22}=\left.\frac{I_{11}}{I_{11} I_{22}-I_{12}^{2}}\right|_{\lambda=\widehat{\lambda}, \gamma=\widehat{\gamma}}
$$

where $\hat{\lambda}$ and $\hat{\gamma}$ are the MLEs of $\lambda$ and $\gamma$, respectively. We will use these asymptotic variances on Section 6 to derive asymptotic confidence intervals for the scale parameter $\lambda$ and the shape parameter $\gamma$.

\section{Simulation Study}

In this section, we assess the performance of the proposed maximum likelihood estimators by a large number of Monte Carlo simulation experiments. All the simulations were carried out in $R$ using the pseudorandom generator in that software package. We generated the progressively Type-II censored samples from the GE-exponential distribution with $\lambda=1.0$ and $\gamma=3.0$ using the algorithm presented by Balakrishnan and Sadhu [19]. The choice of $\gamma=3.0$ provides unimodality and increasing failure rate.

Five values of the sample sizes, say $n=15,20,30,40,50$, different choices of the effective sample size $m$, and different progressive schemes were considered. For brevity in notation, we follow the Balakrishnan, et al. [7] notation to denote the censoring scheme. For example, $(2 * 7,4 * 0)$ denotes the censoring scheme $(7,7,0,0,0,0)$.

We first calculated the moment estimator of the shape parameter $\gamma$ using sample C.V. and Table 1. Then the approximate MLE of the scale parameter $\lambda$ was calculated using (3.14). Approximate MLE of $\gamma$ was then calculated by solving (2.8) numerically. The MLEs of $\lambda$ and 
Table 1: The values of the coefficient of variation (C.V.) for different values of the shape parameter $\gamma$ for the GE-exponential distribution.

\begin{tabular}{|c|c|c|c|c|c|c|c|}
\hline$\underline{r}$ & C.V. & $r$ & C.V. & $r$ & C.V. & $r$ & C.V. \\
\hline 0.1 & 1.847 & 2.2 & 0.825 & 6.5 & 0.642 & 17.0 & 0.522 \\
\hline 0.2 & 1.523 & 2.4 & 0.808 & 7.0 & 0.631 & 18.0 & 0.516 \\
\hline 0.3 & 1.365 & 2.6 & 0.792 & 7.5 & 0.622 & 19.0 & 0.510 \\
\hline 0.4 & 1.265 & 2.8 & 0.779 & 8.0 & 0.613 & 20.0 & 0.505 \\
\hline 0.5 & 1.193 & 3.0 & 0.766 & 8.5 & 0.605 & 21.0 & 0.500 \\
\hline 0.6 & 1.138 & 3.2 & 0.755 & 9.0 & 0.598 & 22.0 & 0.495 \\
\hline 0.7 & 1.094 & 3.4 & 0.744 & 9.5 & 0.591 & 23.0 & 0.491 \\
\hline 0.8 & 1.058 & 3.6 & 0.734 & 10.0 & 0.584 & 24.0 & 0.487 \\
\hline 0.9 & 1.027 & 3.8 & 0.725 & 10.5 & 0.578 & 25.0 & 0.483 \\
\hline 1.0 & 1.000 & 4.0 & 0.717 & 11.0 & 0.572 & 26.0 & 0.479 \\
\hline 1.1 & 0.976 & 4.2 & 0.709 & 11.5 & 0.567 & 27.0 & 0.475 \\
\hline 1.2 & 0.956 & 4.4 & 0.701 & 12.0 & 0.562 & 28.0 & 0.472 \\
\hline 1.3 & 0.937 & 4.6 & 0.694 & 12.5 & 0.557 & 29.0 & 0.468 \\
\hline 1.4 & 0.920 & 4.8 & 0.687 & 13.0 & 0.552 & 30.0 & 0.465 \\
\hline 1.5 & 0.905 & 5.0 & 0.681 & 13.5 & 0.548 & 35.0 & 0.452 \\
\hline 1.6 & 0.890 & 5.2 & 0.675 & 14.0 & 0.544 & 40.0 & 0.440 \\
\hline 1.7 & 0.877 & 5.4 & 0.669 & 14.5 & 0.540 & 45.0 & 0.430 \\
\hline 1.8 & 0.865 & 5.6 & 0.664 & 15.0 & 0.536 & 50.0 & 0.422 \\
\hline 1.9 & 1.854 & 5.8 & 0.659 & 15.5 & 0.532 & 55.0 & 0.414 \\
\hline 2.0 & 1.844 & 6.0 & 0.654 & 16.0 & 0.529 & 60.0 & 0.408 \\
\hline
\end{tabular}

$\gamma$ were calculated by solving the system of normal equations (2.7) and (2.8) simultaneously using BB R package of Varadhan and Gilbert [20]. We repeated this procedure 10000 times.

Tables 2 and 3 give the average of absolute bias, variance, and estimated variance calculated from observed Fisher information for MLEs $\hat{\lambda}$ and $\hat{\gamma}$, respectively. The values of the absolute biases and variances are reasonably small for almost all the sample sizes and censoring schemes indicating the validity of the MLEs $\hat{\lambda}$ and $\hat{\gamma}$. Also, as one would expect, both the bias and the variance decrease as the effective sample proportion that is, $m / n$ increases. Moreover, the observed Fisher information tends to be close to the variance of the estimator for both the MLEs when the effective sample proportion increases.

The tables also show that for all sample sizes $n$ and effective sample sizes $m$, the censoring scheme $R=(n-m, 0, \ldots, 0)$ tends to the smallest bias and variance, whilst the censoring scheme $R=(0,0, \ldots, n-m)$ tends to the largest results. Incidentally, this is in coincidence with the findings of Balakrishnan et al. [9] for Gaussian distribution. 
Table 2: Absolute bias, variance, and variance from observed Fisher information for the MLE of $\lambda$.

\begin{tabular}{|c|c|c|c|c|c|}
\hline$n$ & $m$ & Scheme & $\mathrm{AB}(\widehat{\lambda})$ & $\operatorname{var}(\widehat{\lambda})$ & $\widehat{I}^{11}$ \\
\hline 15 & 5 & $(10,4 * 0)$ & 0.0845 & 0.1124 & 0.1087 \\
\hline 15 & 5 & $(4 * 0,10)$ & 0.0876 & 0.1174 & 0.1113 \\
\hline 15 & 5 & $(5 * 2)$ & 0.0862 & 0.1156 & 0.1145 \\
\hline 15 & 6 & $(9,5 * 0)$ & 0.0745 & 0.0963 & 0.0917 \\
\hline 15 & 6 & $(5 * 0,9)$ & 0.0810 & 0.1035 & 0.0943 \\
\hline 15 & 6 & $(3 * 3,3 * 0)$ & 0.0788 & 0.1012 & 0.0922 \\
\hline 15 & 7 & $(8,6 * 0)$ & 0.0689 & 0.0897 & 0.0842 \\
\hline 15 & 7 & $(6 * 0,8)$ & 0.0736 & 0.0940 & 0.0912 \\
\hline 15 & 7 & $(3 * 0,4 * 2)$ & 0.0755 & 0.0928 & 0.0892 \\
\hline 20 & 6 & $(14,5 * 0)$ & 0.0644 & 0.0912 & 0.0878 \\
\hline 20 & 6 & $(5 * 0,14)$ & 0.0689 & 0.0964 & 0.0912 \\
\hline 20 & 6 & $(2 * 7,4 * 0)$ & 0.0674 & 0.0936 & 0.0890 \\
\hline 20 & 8 & $(12,7 * 0)$ & 0.0574 & 0.0734 & 0.0701 \\
\hline 20 & 8 & $(7 * 0,12)$ & 0.0619 & 0.0792 & 0.0723 \\
\hline 20 & 8 & $(3 * 4,5 * 0)$ & 0.0604 & 0.0756 & 0.0716 \\
\hline 20 & 10 & $(10,9 * 0)$ & 0.0511 & 0.0612 & 0.0579 \\
\hline 20 & 10 & $(9 * 0,10)$ & 0.0556 & 0.0641 & 0.0618 \\
\hline 20 & 10 & $(10 * 1)$ & 0.0544 & 0.0632 & 0.0609 \\
\hline 30 & 8 & $(22,7 * 0)$ & 0.0513 & 0.0667 & 0.0634 \\
\hline 30 & 8 & $(7 * 0,22)$ & 0.0576 & 0.0689 & 0.0655 \\
\hline 30 & 8 & $(8,8,6,5 * 0)$ & 0.0566 & 0.0671 & 0.0640 \\
\hline 30 & 10 & $(20,9 * 0)$ & 0.0409 & 0.0581 & 0.0487 \\
\hline 30 & 10 & $(9 * 0,20)$ & 0.0452 & 0.0621 & 0.0595 \\
\hline 30 & 10 & $(10 * 2)$ & 0.0444 & 0.0604 & 0.0543 \\
\hline 30 & 15 & $(15,14 * 0)$ & 0.0346 & 0.0497 & 0.0442 \\
\hline 30 & 15 & $(14 * 0,15)$ & 0.0380 & 0.0544 & 0.0507 \\
\hline 30 & 15 & $(15 * 1)$ & 0.0354 & 0.0530 & 0.0487 \\
\hline 40 & 15 & $(25,14 * 0)$ & 0.0276 & 0.0482 & 0.0424 \\
\hline 40 & 15 & $(14 * 0,25)$ & 0.0312 & 0.0512 & 0.0464 \\
\hline 40 & 15 & $(10,8,7,12 * 0)$ & 0.0283 & 0.0491 & 0.0448 \\
\hline 40 & 20 & $(20,19 * 0)$ & 0.0214 & 0.0423 & 0.0394 \\
\hline 40 & 20 & $(19 * 0,20)$ & 0.0288 & 0.0449 & 0.0412 \\
\hline 40 & 20 & $(20 * 1)$ & 0.0294 & 0.0433 & 0.0407 \\
\hline 50 & 20 & $(30,19 * 0)$ & 0.0204 & 0.0387 & 0.0325 \\
\hline 50 & 20 & $(19 * 0,30)$ & 0.0298 & 0.0422 & 0.0401 \\
\hline 50 & 20 & $(3 * 10,17 * 0)$ & 0.0254 & 0.0408 & 0.0385 \\
\hline 50 & 25 & $(25,24 * 0)$ & 0.0140 & 0.0319 & 0.0279 \\
\hline 50 & 25 & $(24 * 0,25)$ & 0.0199 & 0.0335 & 0.0308 \\
\hline 50 & 25 & $(25 * 1)$ & 0.0194 & 0.0324 & 0.0309 \\
\hline
\end{tabular}


Table 3: Absolute bias, variance, and variance from observed Fisher information for the MLE of $\gamma$.

\begin{tabular}{|c|c|c|c|c|c|}
\hline$n$ & $m$ & Scheme & $\mathrm{AB}(\widehat{\gamma})$ & $\operatorname{var}(\widehat{\gamma})$ & $\widehat{I}^{22}$ \\
\hline 15 & 5 & $(10,4 * 0)$ & 0.1145 & 0.1328 & 0.1287 \\
\hline 15 & 5 & $(4 * 0,10)$ & 0.1222 & 0.1354 & 0.1279 \\
\hline 15 & 5 & $(5 * 2)$ & 0.1182 & 0.1336 & 0.1259 \\
\hline 15 & 6 & $(9,5 * 0)$ & 0.0954 & 0.1186 & 0.1078 \\
\hline 15 & 6 & $(5 * 0,9)$ & 0.1024 & 0.1245 & 0.1165 \\
\hline 15 & 6 & $(3 * 3,3 * 0)$ & 0.1011 & 0.1200 & 0.1099 \\
\hline 15 & 7 & $(8,6 * 0)$ & 0.0815 & 0.0969 & 0.0888 \\
\hline 15 & 7 & $(6 * 0,8)$ & 0.0878 & 0.1024 & 0.0992 \\
\hline 15 & 7 & $(3 * 0,4 * 2)$ & 0.0855 & 0.0988 & 0.0892 \\
\hline 20 & 6 & $(14,5 * 0)$ & 0.0834 & 0.1012 & 0.0985 \\
\hline 20 & 6 & $(5 * 0,14)$ & 0.0889 & 0.1045 & 0.1028 \\
\hline 20 & 6 & $(2 * 7,4 * 0)$ & 0.0874 & 0.1022 & 0.1001 \\
\hline 20 & 8 & $(12,7 * 0)$ & 0.0712 & 0.0834 & 0.0806 \\
\hline 20 & 8 & $(7 * 0,12)$ & 0.0769 & 0.0912 & 0.0856 \\
\hline 20 & 8 & $(3 * 4,5 * 0)$ & 0.0750 & 0.0878 & 0.0817 \\
\hline 20 & 10 & $(10,9 * 0)$ & 0.0742 & 0.0812 & 0.0785 \\
\hline 20 & 10 & $(9 * 0,10)$ & 0.0785 & 0.0873 & 0.0814 \\
\hline 20 & 10 & $(10 * 1)$ & 0.0766 & 0.0832 & 0.0804 \\
\hline 30 & 8 & $(22,7 * 0)$ & 0.0643 & 0.0912 & 0.0825 \\
\hline 30 & 8 & $(7 * 0,22)$ & 0.0692 & 0.0945 & 0.0854 \\
\hline 30 & 8 & $(8,8,6,5 * 0)$ & 0.0664 & 0.0842 & 0.0833 \\
\hline 30 & 10 & $(20,9 * 0)$ & 0.0546 & 0.0722 & 0.0689 \\
\hline 30 & 10 & $(9 * 0,20)$ & 0.0612 & 0.0780 & 0.0729 \\
\hline 30 & 10 & $(10 * 2)$ & 0.0594 & 0.0755 & 0.0680 \\
\hline 30 & 15 & $(15,14 * 0)$ & 0.0392 & 0.0571 & 0.0536 \\
\hline 30 & 15 & $(14 * 0,15)$ & 0.0472 & 0.0633 & 0.0582 \\
\hline 30 & 15 & $(15 * 1)$ & 0.0469 & 0.0627 & 0.0573 \\
\hline 40 & 15 & $(25,14 * 0)$ & 0.0326 & 0.0574 & 0.0489 \\
\hline 40 & 15 & $(14 * 0,25)$ & 0.0385 & 0.0595 & 0.0536 \\
\hline 40 & 15 & $(10,8,7,12 * 0)$ & 0.0342 & 0.0570 & 0.0569 \\
\hline 40 & 20 & $(20,19 * 0)$ & 0.0214 & 0.0423 & 0.0394 \\
\hline 40 & 20 & $(19 * 0,20)$ & 0.0288 & 0.0449 & 0.0412 \\
\hline 40 & 20 & $(20 * 1)$ & 0.0294 & 0.0433 & 0.0407 \\
\hline 50 & 20 & $(30,19 * 0)$ & 0.0279 & 0.0457 & 0.0398 \\
\hline 50 & 20 & $(19 * 0,30)$ & 0.0356 & 0.0482 & 0.0441 \\
\hline 50 & 20 & $(3 * 10,17 * 0)$ & 0.0254 & 0.0408 & 0.0385 \\
\hline 50 & 25 & $(25,24 * 0)$ & 0.0289 & 0.0387 & 0.0356 \\
\hline 50 & 25 & $(24 * 0,25)$ & 0.0260 & 0.0415 & 0.0388 \\
\hline 50 & 25 & $(25 * 1)$ & 0.0289 & 0.0393 & 0.0370 \\
\hline
\end{tabular}


Table 4: 90\% (First column) and 95\% (Second column) coverage probabilities for the parameters $\lambda$ and $\gamma$.

\begin{tabular}{|c|c|c|c|c|c|c|}
\hline$n$ & $m$ & Scheme & & & & \\
\hline 15 & 5 & $(10,4 * 0)$ & 0.9089 & 0.9406 & 0.8612 & 0.9035 \\
\hline 15 & 5 & $(4 * 0,10)$ & 0.9074 & 0.9523 & 0.8642 & 0.9042 \\
\hline 15 & 5 & $(5 * 2)$ & 0.9027 & 0.9504 & 0.8781 & 0.9089 \\
\hline 15 & 6 & $(9,5 * 0)$ & 0.9086 & 0.9512 & 0.8697 & 0.9125 \\
\hline 15 & 6 & $(5 * 0,9)$ & 0.9108 & 0.9508 & 0.8723 & 0.9154 \\
\hline 15 & 6 & $(3 * 3,3 * 0)$ & 0.9084 & 0.9521 & 0.8706 & 0.9123 \\
\hline 15 & 7 & $(8,6 * 0)$ & 0.9145 & 0.9571 & 0.8785 & 0.9241 \\
\hline 15 & 7 & $(6 * 0,8)$ & 0.9092 & 0.9574 & 0.8717 & 0.9224 \\
\hline 15 & 7 & $(3 * 0,4 * 2)$ & 0.9071 & 0.9514 & 0.8792 & 0.9235 \\
\hline 20 & 6 & $(14,5 * 0)$ & 0.9136 & 0.9571 & 0.8714 & 0.9106 \\
\hline 20 & 6 & $(5 * 0,14)$ & 0.9074 & 0.9583 & 0.8628 & 0.9164 \\
\hline 20 & 6 & $(2 * 7,4 * 0)$ & 0.9047 & 0.9538 & 0.8705 & 0.9188 \\
\hline 20 & 8 & $(12,7 * 0)$ & 0.9068 & 0.9579 & 0.8752 & 0.9178 \\
\hline 20 & 8 & $(7 * 0,12)$ & 0.9008 & 0.9479 & 0.8666 & 0.9232 \\
\hline 20 & 8 & $(3 * 4,5 * 0)$ & 0.9064 & 0.9587 & 0.8661 & 0.9213 \\
\hline 20 & 10 & $(10,9 * 0)$ & 0.9034 & 0.9513 & 0.8886 & 0.9274 \\
\hline 20 & 10 & $(9 * 0,10)$ & 0.9063 & 0.9517 & 0.8708 & 0.9341 \\
\hline 20 & 10 & $(10 * 1)$ & 0.9025 & 0.9516 & 0.8841 & 0.9378 \\
\hline 30 & 8 & $(22,7 * 0)$ & 0.9187 & 0.9574 & 0.8706 & 0.9047 \\
\hline 30 & 8 & $(7 * 0,22)$ & 0.9023 & 0.9508 & 0.8678 & 0.9314 \\
\hline 30 & 8 & $(8,8,6,5 * 0)$ & 0.9014 & 0.9614 & 0.8617 & 0.9264 \\
\hline 30 & 10 & $(20,9 * 0)$ & 0.9035 & 0.9531 & 0.8756 & 0.9218 \\
\hline 30 & 10 & $(9 * 0,20)$ & 0.9098 & 0.9618 & 0.8707 & 0.9246 \\
\hline 30 & 10 & $(10 * 2)$ & 0.9059 & 0.9503 & 0.8713 & 0.9279 \\
\hline 30 & 15 & $(15,14 * 0)$ & 0.9081 & 0.9530 & 0.8966 & 0.9308 \\
\hline 30 & 15 & $(14 * 0,15)$ & 0.9146 & 0.9618 & 0.8942 & 0.9446 \\
\hline 30 & 15 & $(15 * 1)$ & 0.9042 & 0.9589 & 0.8914 & 0.9446 \\
\hline 40 & 15 & $(25,14 * 0)$ & 0.9184 & 0.9564 & 0.8813 & 0.9324 \\
\hline 40 & 15 & $(14 * 0,25)$ & 0.9016 & 0.9508 & 0.8863 & 0.9474 \\
\hline 40 & 15 & $(10,8,7,12 * 0)$ & 0.9023 & 0.9574 & 0.8848 & 0.9358 \\
\hline 40 & 20 & $(20,19 * 0)$ & 0.9065 & 0.9574 & 0.8965 & 0.9345 \\
\hline 40 & 20 & $(19 * 0,20)$ & 0.9082 & 0.9549 & 0.8823 & 0.9349 \\
\hline 40 & 20 & $(20 * 1)$ & 0.9028 & 0.9526 & 0.8754 & 0.9377 \\
\hline 50 & 20 & $(30,19 * 0)$ & 0.9048 & 0.9508 & 0.8685 & 0.9305 \\
\hline 50 & 20 & $(19 * 0,30)$ & 0.9092 & 0.9534 & 0.8872 & 0.9375 \\
\hline 50 & 20 & $(3 * 10,17 * 0)$ & 0.9047 & 0.9549 & 0.8863 & 0.9347 \\
\hline 50 & 25 & $(25,24 * 0)$ & 0.9145 & 0.9541 & 0.8968 & 0.9417 \\
\hline 50 & 25 & $(24 * 0,25)$ & 0.9095 & 0.9549 & 0.8927 & 0.9429 \\
\hline 50 & 25 & $(25 * 1)$ & 0.9080 & 0.9564 & 0.8933 & 0.9448 \\
\hline
\end{tabular}


Table 5: (5.0, 95.0) (First column) and $(2.5,97.5)$ (Second column) percentage points for the pivotal quantities.

\begin{tabular}{|c|c|c|c|c|c|c|}
\hline$n$ & $m$ & Scheme & & & & \\
\hline 15 & 5 & $(10,4 * 0)$ & $(-1.54,1.61)$ & $(-1.94,1.85)$ & $(-1.08,2.65)$ & $(-1.24,3.39)$ \\
\hline 15 & 5 & $(4 * 0,10)$ & $(-1.73,1.52)$ & $(-2.18,1.75)$ & $(-1.64,2.12)$ & $(-2.00,2.63)$ \\
\hline 15 & 5 & $(5 * 2)$ & $(-1.59,1.62)$ & $(-2.00,1.85)$ & $(-1.22,2.48)$ & $(-1.44,3.13)$ \\
\hline 15 & 6 & $(9,5 * 0)$ & $(-1.56,1.63)$ & $(-1.94,1.89)$ & $(-1.18,2.44)$ & $(-1.38,3.08)$ \\
\hline 15 & 6 & $(5 * 0,9)$ & $(-1.70,1.54)$ & $(-2.15,1.77)$ & $(-1.64,2.04)$ & $(-1.99,2.53)$ \\
\hline 15 & 6 & $(3 * 3,3 * 0)$ & $(-1.68,1.55)$ & $(-2.11,1.78)$ & $(-1.58,2.12)$ & $(-1.91,2.61)$ \\
\hline 15 & 7 & $(8,6 * 0)$ & $(-1.58,1.63)$ & $(-1.93,1.88)$ & $(-1.26,2.77)$ & $(-1.48,2.82)$ \\
\hline 15 & 7 & $(6 * 0,8)$ & $(-1.72,1.52)$ & $(-2.12,1.77)$ & $(-1.66,1.94)$ & $(-1.99,2.42)$ \\
\hline 15 & 7 & $(3 * 0,4 * 2)$ & $(-1.61,1.62)$ & $(-1.97,1.87)$ & $(-1.32,2.22)$ & $(-1.55,2.75)$ \\
\hline 20 & 6 & $(14,5 * 0)$ & $(-1.55,1.61)$ & $(-1.93,1.85)$ & $(-1.07,2.59)$ & $(-1.21,3.27)$ \\
\hline 20 & 6 & $(5 * 0,14)$ & $(-1.70,1.54)$ & $(-2.15,1.77)$ & $(-1.62,2.05)$ & $(-1.98,2.54)$ \\
\hline 20 & 6 & $(2 * 7,4 * 0)$ & $(-1.70,1.54)$ & $(-2.14,1.76)$ & $(-1.59,2.10)$ & $(-1.90,2.59)$ \\
\hline 20 & 8 & $(12,7 * 0)$ & $(-1.57,1.64)$ & $(-1.93,1.88)$ & $(-1.23,2.31)$ & $(-1.44,2.80)$ \\
\hline 20 & 8 & $(7 * 0,12)$ & $(-1.73,1.55)$ & $(-2.12,1.78)$ & $(-1.64,1.95)$ & $(-1.97,2.36)$ \\
\hline 20 & 8 & $(3 * 4,5 * 0)$ & $(-1.71,1.56)$ & $(-2.10,1.78)$ & $(-1.60,1.99)$ & $(-1.92,2.42)$ \\
\hline 20 & 10 & $(10,9 * 0)$ & $(-1.59,1.65)$ & $(-1.96,1.91)$ & $(-1.33,2.15)$ & $(-1.58,2.60)$ \\
\hline 20 & 10 & $(9 * 0,10)$ & $(-1.70,1.56)$ & $(-2.12,1.81)$ & $(-1.64,1.90)$ & $(-1.97,2.31)$ \\
\hline 20 & 10 & $(10 * 1)$ & $(-1.63,1.62)$ & $(-2.01,1.87)$ & $(-1.45,2.04)$ & $(-1.72,2.48)$ \\
\hline 30 & 8 & $(22,7 * 0)$ & $(-1.56,1.62)$ & $(-1.93,1.83)$ & $(-1.08,2.48)$ & $(-1.22,3.01)$ \\
\hline 30 & 8 & $(7 * 0,22)$ & $(-1.73,1.55)$ & $(-2.12,1.78)$ & $(-1.64,1.96)$ & $(-1.96,2.38)$ \\
\hline 30 & 8 & $(8,8,6,5 * 0)$ & $(-1.71,1.55)$ & $(-2.11,1.77)$ & $(-1.57,2.03)$ & $(-1.89,2.47)$ \\
\hline 30 & 10 & $(20,9 * 0)$ & $(-1.57,1.64)$ & $(-1.93,1.89)$ & $(-1.20,2.30)$ & $(-1.40,2.81)$ \\
\hline 30 & 10 & $(9 * 0,20)$ & $(-1.71,1.56)$ & $(-2.13,1.81)$ & $(-1.64,1.91)$ & $(-1.97,2.32)$ \\
\hline 30 & 10 & $(10 * 2)$ & $(-1.61,1.64)$ & $(-1.97,1.88)$ & $(-1.32,2.17)$ & $(-1.56,2.63)$ \\
\hline 30 & 15 & $(15,14 * 0)$ & $(-1.57,1.64)$ & $(-1.89,1.92)$ & $(-1.35,2.01)$ & $(-1.58,2.44)$ \\
\hline 30 & 15 & $(14 * 0,15)$ & $(-1.68,1.56)$ & $(-1.99,1.84)$ & $(-1.62,1.80)$ & $(-1.89,2.19)$ \\
\hline 30 & 15 & $(15 * 1)$ & $(-1.62,1.61)$ & $(-1.95,1.87)$ & $(-1.46,1.93)$ & $(-1.72,2.34)$ \\
\hline 40 & 15 & $(25,14 * 0)$ & $(-1.56,1.64)$ & $(-1.87,1.91)$ & $(-1.29,2.10)$ & $(-1.49,2.55)$ \\
\hline 40 & 15 & $(14 * 0,25)$ & $(-1.68,1.56)$ & $(-1.99,1.83)$ & $(-1.62,1.80)$ & $(-1.88,2.19)$ \\
\hline 40 & 15 & $(10,8,7,12 * 0)$ & $(-1.68,1.56)$ & $(-1.99,1.83)$ & $(-1.61,1.83)$ & $(-1.88,2.21)$ \\
\hline 40 & 20 & $(20,19 * 0)$ & $(-1.61,1.66)$ & $(-1.97,1.92)$ & $(-1.41,1.98)$ & $(-1.68,2.37)$ \\
\hline 40 & 20 & $(19 * 0,20)$ & $(-1.69,1.59)$ & $(-2.05,1.86)$ & $(-1.63,1.80)$ & $(-1.95,2.15)$ \\
\hline 40 & 20 & $(20 * 1)$ & $(-1.64,1.63)$ & $(-2.02,1.89)$ & $(-1.51,1.90)$ & $(-1.80,2.27)$ \\
\hline 50 & 20 & $(30,19 * 0)$ & $(-1.60,1.67)$ & $(-1.95,1.92)$ & $(-1.35,2.04)$ & $(-1.60,2.44)$ \\
\hline 50 & 20 & $(19 * 0,30)$ & $(-1.69,1.59)$ & $(-2.05,1.86)$ & $(-1.63,1.80)$ & $(-1.95,2.16)$ \\
\hline 50 & 20 & $(3 * 10,17 * 0)$ & $(-1.70,1.59)$ & $(-2.05,1.85)$ & $(-1.62,1.82)$ & $(-1.95,2.17)$ \\
\hline 50 & 25 & $(25,24 * 0)$ & $(-1.61,1.63)$ & $(-1.94,1.92)$ & $(-1.43,1.90)$ & $(-1.68,2.30)$ \\
\hline 50 & 25 & $(24 * 0,25)$ & $(-1.68,1.58)$ & $(-2.04,1.84)$ & $(-1.63,1.75)$ & $(-1.93,2.09)$ \\
\hline 50 & 25 & $(25 * 1)$ & $(-1.64,1.61)$ & $(-1.97,1.88)$ & $(-1.52,1.83)$ & $(-1.79,2.21)$ \\
\hline
\end{tabular}


Table 6: Ball bearings data.

\begin{tabular}{lcccccccr}
\hline 17.88 & 28.92 & 33.00 & 41.52 & 42.12 & 45.60 & 48.48 & 51.84 & 51.96 \\
54.12 & 55.56 & 67.80 & $67.80^{*}$ & $67.80^{*}$ & 68.64 & $68.64^{*}$ & $68.68^{*}$ & 84.12 \\
93.12 & 98.64 & 105.12 & $105.84^{*}$ & 127.92 & 128.04 & $173.40^{*}$ & & \\
\hline
\end{tabular}

\section{Asymptotic Confidence Intervals}

In this section, we study the asymptotic confidence intervals for the parameters $\lambda$ and $\gamma$ using the estimation for the observed Fisher information matrix. Note that the asymptotic distributions of the pivotal quantities

$$
P_{1}=\frac{\widehat{\imath}-\lambda}{\sqrt{\widehat{I}^{11}}}, \quad P_{2}=\frac{\widehat{\gamma}-\gamma}{\sqrt{\widehat{I}^{22}}}
$$

are standard normal distribution. The asymptotic $100(1-\alpha) \%$ confidence intervals for the parameters are then given by

$$
\widehat{\lambda} \pm z_{\alpha / 2} \sqrt{\widehat{I}^{11}}, \quad \widehat{\gamma} \pm z_{\alpha / 2} \sqrt{\widehat{I}^{22}}
$$

where $z_{\alpha / 2}$ is the upper $\alpha / 2$ critical value of the standard normal distribution.

Table 4 provides the coverage probabilities through 10000 Monte Carlo simulations for the parameters $\lambda$ and $\gamma$. It is observed that the coverage probabilities are very close to their nominated values even for small sample sizes for both confidence intervals.

We also calculated the unconditional percentage points of these pivotal quantities and the results are given in Table 5. The values of the percentage points associate with $P_{1}$ and $P_{2}$ support the standard normality of these pivotal quantities specifically for large values of the sample size and the effective sample proportion. Indeed, the percentage points in Table 5 could be used to construct another asymptotic confidence intervals for the unknown parameters.

\section{Real Data Analysis}

In this section, we consider a real dataset to illustrate the inferential procedure developed in this article. The data studied by various authors are the number of the million revolutions before failure of the 25 ball bearings provided by Lieblein and Zelen [21]. The data are one of the most frequently used datasets in literature although incorrectly as a complete dataset, however, the data are indeed censored (see [22]).

Table 6 shows the ball bearings data. The six data values, as marked with asterisks, were discarded from analysis (censored). In Table 7, we presented the censored data as well as the progressively Type-II censoring scheme.

The sample C.V. of the data is found to be 0.4917 and Table 1 suggests the value of the moment estimator of the shape parameter to be $\tilde{\gamma}=23$.

We found the approximate estimators for the parameters to be $\breve{\lambda}=0.0410$ and $\breve{\gamma}=$ 19.1628. These estimates were then used as a starting values to find the MLEs from the system 
Table 7: Ball bearings data and progressively Type II censoring scheme.

\begin{tabular}{lcccccccccc}
\hline$i$ & 1 & 2 & 3 & 4 & 5 & 6 & 7 & 8 & 9 & 10 \\
\hline$x_{i: m: n}$ & 17.88 & 28.92 & 33.00 & 41.52 & 42.12 & 45.60 & 48.48 & 51.84 & 51.96 & 54.12 \\
$R_{i}$ & 0 & 0 & 0 & 0 & 0 & 0 & 0 & 0 & 0 & 0 \\
\hline$i$ & 11 & 12 & 13 & 14 & 15 & 16 & 17 & 18 & 19 \\
\hline$x_{i: m: n}$ & 55.56 & 67.80 & 68.64 & 84.12 & 93.12 & 98.64 & 105.12 & 127.92 & 128.04 \\
$R_{i}$ & 2 & 0 & 2 & 0 & 0 & 0 & 1 & 0 & 1 \\
\hline
\end{tabular}

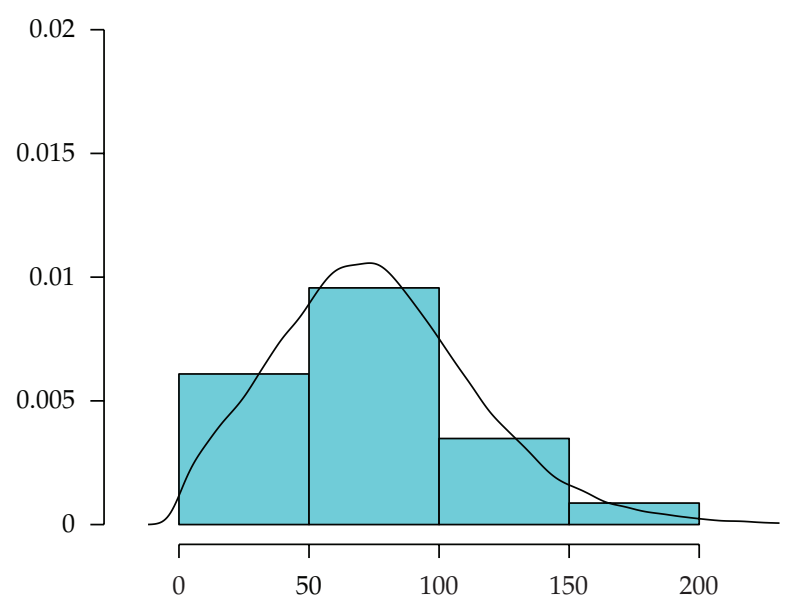

Figure 1: Histogram of the ball bearings data and the density function of the fitted GE-exponential model.

of normal equations (2.7) and (2.8) numerically. We obtained $\hat{\lambda}=0.04$ and $\hat{\gamma}=17.67$. Figure 1 depicts the histogram of the data and the fitted GE-exponential $(0.04,17.67)$.

\section{References}

[1] R. G. Herd, Estimation of the parameters of a population from a multi-censored sample [Ph.D. thesis], Iowa State College, Ames, Iowa, USA, 1956.

[2] A. C. Cohen," "Progressively censored samples in life testing," Technometrics, vol. 5, pp. 327-339, 1963.

[3] N. Balakrishnan and R. Aggrawala, Progressive Censoring: Theory, Methods, and Applications, Birkhäauser, Boston, Mass, USA, 2000.

[4] N. Balakrishnan, "Progressive censoring methodology: an appraisal," TEST, vol. 16, no. 2, pp. 211$259,2007$.

[5] N. R. Mann, "Exact three-order statistic confidence bounds on reliable life for a Weibull model with progressive censoring," Journal of the American Statistical Association, vol. 64, pp. 306-315, 1969.

[6] N. R. Mann, "Best linear invariant estimation for Weibull parameters under progressive censoring," Technometrics, vol. 13, pp. 521-533, 1971.

[7] N. Balakrishnan, N. Kannan, C. T. Lin, and S. J. S. Wu, "Inference for the extreme value distribution under progressive Type-II censoring," Journal of Statistical Computation and Simulation, vol. 74, no. 1, pp. 25-45, 2004.

[8] N. Balakrishnan and N. Kannan, "Point and interval estimation for the parameters of the logistic distribution based on progressively Type-II censored samples," in Handbook of Statistics, N. Balakrishnan and C. R. Rao, Eds., vol. 20, pp. 431-456, 2000.

[9] N. Balakrishnan, N. Kannan, C. T. Lin, and H. K. T. Ng, "Point and interval estimation for Gaussian distribution, based on progressively Type-II censored samples," Journal of Statistical Computation and Simulation, vol. 74, pp. 25-45, 2003. 
[10] C. Kim and K. Han, "Estimation of the scale parameter of the half-logistic distribution under progressively Type-II censored sample," Statistical Papers, vol. 51, no. 2, pp. 375-387, 2010.

[11] C.-T. Lin, S. J. S. Wu, and N. Balakrishnan, "Inference for log-gamma distribution based on progressively Type-II censored data," Communications in Statistics, vol. 35, no. 7-9, pp. 1271-1292, 2006.

[12] A. Asgharzadeh, "Approximate MLE for the scaled generalized exponential distribution under progressive Type-II censoring," Journal of the Korean Statistical Society, vol. 38, no. 3, pp. 223-229, 2009.

[13] A. W. Marshall and I. Olkin, "A new method for adding a parameter to a family of distributions with application to the exponential and Weibull families," Biometrika, vol. 84, no. 3, pp. 641-652, 1997.

[14] K. Adamidis and S. Loukas, "A lifetime distribution with decreasing failure rate," Statistics $\mathcal{E}$ Probability Letters, vol. 39, no. 1, pp. 35-42, 1998.

[15] K. Adamidis, T. Dimitrakopoulou, and S. Loukas, "On an extension of the exponential-geometric distribution," Statistics E Probability Letters, vol. 73, no. 3, pp. 259-269, 2005.

[16] A. W. Marshall, J. C. Meza, and I. Olkin, “Can data recognize its parent distribution?" Journal of Computational and Graphical Statistics, vol. 10, no. 3, pp. 555-580, 2001.

[17] R. Pakyari, "Discriminating between generalized exponential, geometric extreme exponential and Weibull distributions," Journal of Statistical Computation and Simulation, vol. 80, no. 12, pp. 1403-1412, 2010.

[18] A. Erdeli, W. Magnus, F. Oberhettinger, and F. G. Tricomi, Higher Transcendental Functions, McGrawHill, New York, NY, USA, 1953.

[19] N. Balakrishnan and R. A. Sandhu, "A simple simulational algorithm for generating progressive Type-II censored samples," The American Satistican, vol. 49, no. 2, pp. 229-230, 1995.

[20] R. Varadhan and P. D. Gilbert, "BB: an R package for solving a large system of nonlinear equations and for optimizing a high-dimensional nonlinear objective function," Journal of Statistical Software, vol. 32, no. 4, pp. 1-26, 2009.

[21] J. Lieblein and M. Zelen, "Statistical investigation of the fatigue life of deep-groove ball bearings," Journal of Research of the National Bureau of Standards, vol. 57, pp. 273-316, 1956.

[22] C. Caroni, "The correct "ball bearings" data," Lifetime Data Analysis, vol. 8, no. 4, pp. 395-399, 2002. 


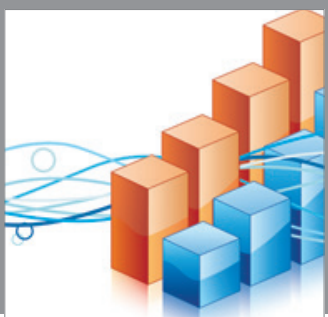

Advances in

Operations Research

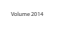

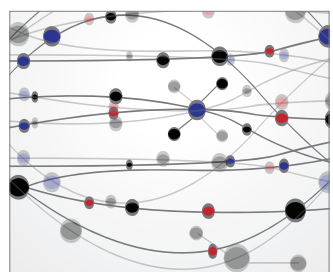

\section{The Scientific} World Journal
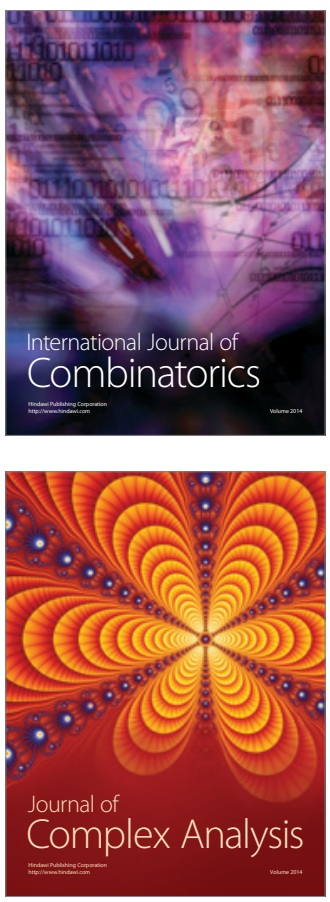

International Journal of

Mathematics and

Mathematical

Sciences
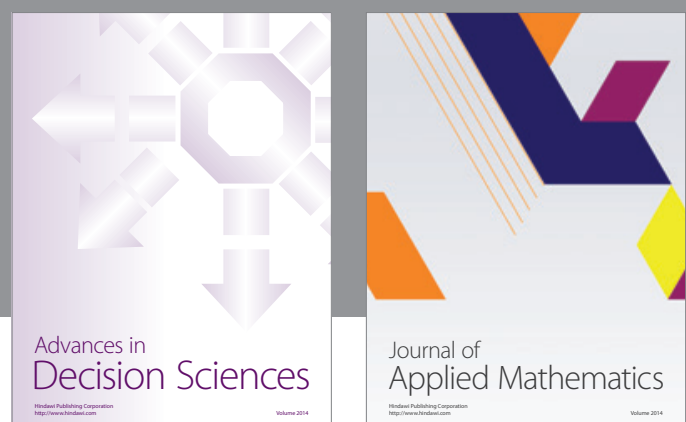

Journal of

Applied Mathematics
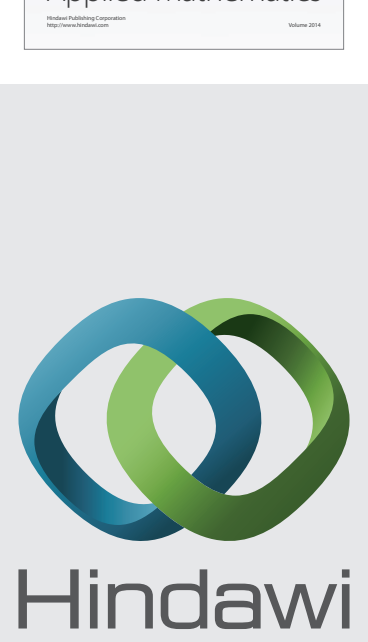

Submit your manuscripts at http://www.hindawi.com
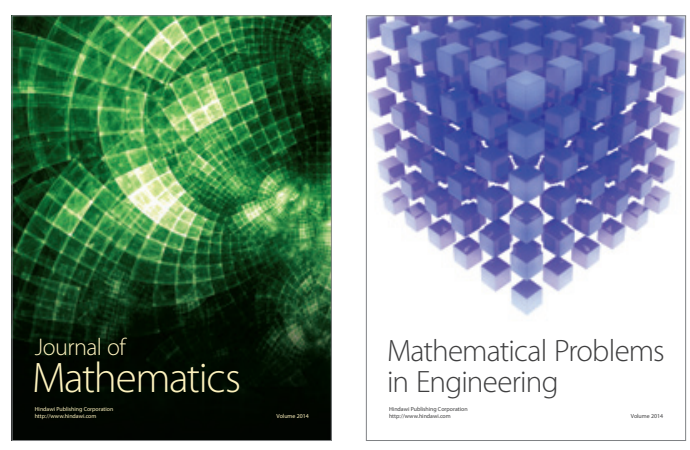

Mathematical Problems in Engineering
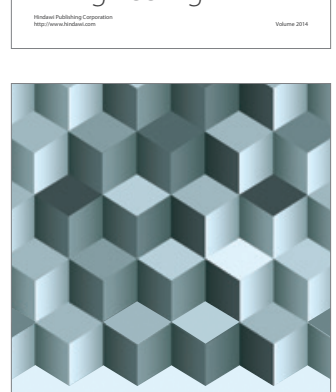

Journal of

Function Spaces
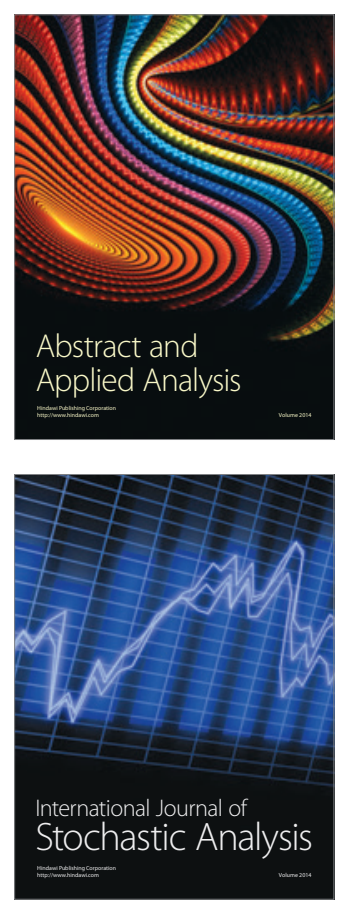

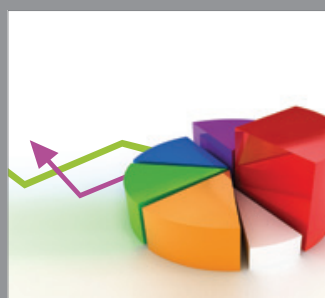

ournal of

Probability and Statistics

Promensencen
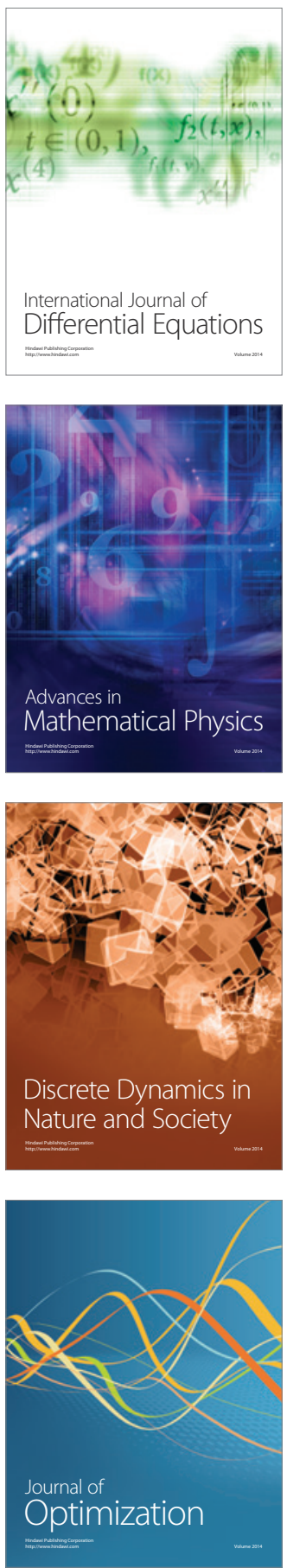